\title{
Heat Flow, Work Energy, Chemical Reactions, and Thermodynamics: A Dynamical Systems Perspective
}

\author{
Wassim M. Haddad ${ }^{1}$, Sergey G. Nersesov ${ }^{2}$ and VijaySekhar Chellaboina ${ }^{3}$ \\ ${ }^{1}$ Georgia Institute of Technology \\ ${ }^{2}$ Villanova University \\ ${ }^{3}$ Tata Consultancy Services \\ ${ }^{1,2}$ USA \\ ${ }^{3}$ INDIA
}

\section{Introduction}

There is no doubt that thermodynamics is a theory of universal proportions whose laws reign supreme among the laws of nature and are capable of addressing some of science's most intriguing questions about the origins and fabric of our universe. The laws of thermodynamics are among the most firmly established laws of nature and play a critical role in the understanding of our expanding universe. In addition, thermodynamics forms the underpinning of several fundamental life science and engineering disciplines, including biological systems, physiological systems, chemical reaction systems, ecological systems, information systems, and network systems, to cite but a few examples. While from its inception its speculations about the universe have been grandiose, its mathematical foundation has been amazingly obscure and imprecise (Truesdell (1969; 1980); Arnold (1990); Haddad et al. (2005)). This is largely due to the fact that classical thermodynamics is a physical theory concerned mainly with equilibrium states and does not possess equations of motion. The absence of a state space formalism in classical thermodynamics, and physics in general, is quite disturbing and in our view largely responsible for the monomeric state of classical thermodynamics.

In recent research, Haddad et al. (2005; 2008) combined the two universalisms of thermodynamics and dynamical systems theory under a single umbrella to develop a dynamical system formalism for classical thermodynamics so as to harmonize it with classical mechanics. While it seems impossible to reduce thermodynamics to a mechanistic world picture due to microscopic reversibility and Poincaré recurrence, the system thermodynamic formulation of Haddad et al. (2005) provides a harmonization of classical thermodynamics with classical mechanics. In particular, our dynamical system formalism captures all of the key aspects of thermodynamics, including its fundamental laws, while providing a mathematically rigorous formulation for thermodynamical systems out of equilibrium by unifying the theory of heat transfer with that of classical thermodynamics. In addition, the concept of entropy for a nonequilibrium state of a dynamical process is defined, and its global existence and uniqueness is established. This state space formalism of thermodynamics shows 
that the behavior of heat, as described by the conservation equations of thermal transport and as described by classical thermodynamics, can be derived from the same basic principles and is part of the same scientific discipline. Connections between irreversibility, the second law of thermodynamics, and the entropic arrow of time are also established in Haddad et al. (2005). Specifically, we show a state irrecoverability and, hence, a state irreversibility nature of thermodynamics. State irreversibility reflects time-reversal non-invariance, wherein time-reversal is not meant literally; that is, we consider dynamical systems whose trajectory reversal is or is not allowed and not a reversal of time itself. In addition, we show that for every nonequilibrium system state and corresponding system trajectory of our thermodynamically consistent dynamical system, there does not exist a state such that the corresponding system trajectory completely recovers the initial system state of the dynamical system and at the same time restores the energy supplied by the environment back to its original condition. This, along with the existence of a global strictly increasing entropy function on every nontrivial system trajectory, establishes the existence of a completely ordered time set having a topological structure involving a closed set homeomorphic to the real line giving a clear time-reversal asymmetry characterization of thermodynamics and establishing an emergence of the direction of time flow.

In this paper, we reformulate and extend some of the results of Haddad et al. (2005). In particular, unlike the framework in Haddad et al. (2005) wherein we establish the existence and uniqueness of a global entropy function of a specific form for our thermodynamically consistent system model, in this paper we assume the existence of a continuously differentiable, strictly concave function that leads to an entropy inequality that can be identified with the second law of thermodynamics as a statement about entropy increase. We then turn our attention to stability and convergence. Specifically, using Lyapunov stability theory and the Krasovskii-LaSalle invariance principle, we show that for an adiabatically isolated system the proposed interconnected dynamical system model is Lyapunov stable with convergent trajectories to equilibrium states where the temperatures of all subsystems are equal. Finally, we present a state-space dynamical system model for chemical thermodynamics. In particular, we use the law of mass-action to obtain the dynamics of chemical reaction networks. Furthermore, using the notion of the chemical potential (Gibbs $(1875 ; 1878))$, we unify our state space mass-action kinetics model with our thermodynamic dynamical system model involving energy exchange. In addition, we show that entropy production during chemical reactions is nonnegative and the dynamical system states of our chemical thermodynamic state space model converge to a state of temperature equipartition and zero affinity (i.e., the difference between the chemical potential of the reactants and the chemical potential of the products in a chemical reaction).

\section{Mathematical preliminaries}

In this section, we establish notation, definitions, and provide some key results necessary for developing the main results of this paper. Specifically, $\mathbb{R}$ denotes the set of real numbers, $\overline{\mathbb{Z}}_{+}$ (respectively, $\mathbb{Z}_{+}$) denotes the set of nonnegative (respectively, positive) integers, $\mathbb{R}^{q}$ denotes the set of $q \times 1$ column vectors, $\mathbb{R}^{n \times m}$ denotes the set of $n \times m$ real matrices, $\mathbb{P}^{n}$ (respectively, $\mathbb{N}^{n}$ ) denotes the set of positive (respectively, nonnegative) definite matrices, $(\cdot)^{\mathrm{T}}$ denotes transpose, $I_{q}$ or $I$ denotes the $q \times q$ identity matrix, e denotes the ones vector of order $q$, that is, $\mathbf{e} \triangleq[1, \ldots, 1]^{\mathrm{T}} \in \mathbb{R}^{q}$, and $\mathbf{e}_{i} \in \mathbb{R}^{q}$ denotes a vector with unity in the $i$ th component and zeros elsewhere. For $x \in \mathbb{R}^{q}$ we write $x \geq \geq 0$ (respectively, $x>>0$ ) to indicate that every component of $x$ is nonnegative (respectively, positive). In this case, we say that $x$ is 
nonnegative or positive, respectively. Furthermore, $\overline{\mathbb{R}}_{+}^{q}$ and $\mathbb{R}_{+}^{q}$ denote the nonnegative and positive orthants of $\mathbb{R}^{q}$, that is, if $x \in \mathbb{R}^{q}$, then $x \in \overline{\mathbb{R}}_{+}^{q}$ and $x \in \mathbb{R}_{+}^{q}$ are equivalent, respectively, to $x \geq \geq 0$ and $x>>0$. Analogously, $\overline{\mathbb{R}}_{+}^{n \times m}$ (respectively, $\mathbb{R}_{+}^{n \times m}$ ) denotes the set of $n \times m$ real matrices whose entries are nonnegative (respectively, positive). For vectors $x, y \in \mathbb{R}^{q}$, with components $x_{i}$ and $y_{i}, i=1, \ldots, q$, we use $x \circ y$ to denote component-by-component multiplication, that is, $x \circ y \triangleq\left[x_{1} y_{1}, \ldots, x_{q} y_{q}\right]^{\mathrm{T}}$. Finally, we write $\partial \mathcal{S}, \stackrel{\circ}{\mathcal{S}}$, and $\overline{\mathcal{S}}$ to denote the boundary, the interior, and the closure of the set $\mathcal{S}$, respectively.

We write $\|\cdot\|$ for the Euclidean vector norm, $V^{\prime}(x) \triangleq \frac{\partial V(x)}{\partial x}$ for the Fréchet derivative of $V$ at $x, \mathcal{B}_{\varepsilon}(\alpha), \alpha \in \mathbb{R}^{q}, \varepsilon>0$, for the open ball centered at $\alpha$ with radius $\varepsilon$, and $x(t) \rightarrow \mathcal{M}$ as $t \rightarrow \infty$ to denote that $x(t)$ approaches the set $\mathcal{M}$ (that is, for every $\varepsilon>0$ there exists $T>0$ such that $\operatorname{dist}(x(t), \mathcal{M})<\varepsilon$ for all $t>T$, where $\left.\operatorname{dist}(p, \mathcal{M}) \triangleq \inf _{x \in \mathcal{M}}\|p-x\|\right)$. The notions of openness, convergence, continuity, and compactness that we use throughout the paper refer to the topology generated on $\mathcal{D} \subseteq \mathbb{R}^{q}$ by the norm $\|\cdot\|$. A subset $\mathcal{N}$ of $\mathcal{D}$ is relatively open in $\mathcal{D}$ if $\mathcal{N}$ is open in the subspace topology induced on $\mathcal{D}$ by the norm $\|\cdot\|$. A point $x \in \mathbb{R}^{q}$ is a subsequential limit of the sequence $\left\{x_{i}\right\}_{i=0}^{\infty}$ in $\mathbb{R}^{q}$ if there exists a subsequence of $\left\{x_{i}\right\}_{i=0}^{\infty}$ that converges to $x$ in the norm $\|\cdot\|$. Recall that every bounded sequence has at least one subsequential limit. A divergent sequence is a sequence having no convergent subsequence.

Consider the nonlinear autonomous dynamical system

$$
\dot{x}(t)=f(x(t)), \quad x(0)=x_{0}, \quad t \in \mathcal{I}_{x_{0}},
$$

where $x(t) \in \mathcal{D} \subseteq \mathbb{R}^{n}, t \in \mathcal{I}_{x_{0}}$, is the system state vector, $\mathcal{D}$ is a relatively open set, $f: \mathcal{D} \rightarrow \mathbb{R}^{n}$ is continuous on $\mathcal{D}$, and $\mathcal{I}_{x_{0}}=\left[0, \tau_{x_{0}}\right), 0 \leq \tau_{x_{0}} \leq \infty$, is the maximal interval of existence for the solution $x(\cdot)$ of (1). We assume that, for every initial condition $x(0) \in \mathcal{D}$, the differential equation (1) possesses a unique right-maximally defined continuously differentiable solution which is defined on $[0, \infty)$. Letting $s(\cdot, x)$ denote the right-maximally defined solution of (1) that satisfies the initial condition $x(0)=x$, the above assumptions imply that the map $s:[0, \infty) \times \mathcal{D} \rightarrow \mathcal{D}$ is continuous (Hartman, 1982, Theorem V.2.1), satisfies the consistency property $s(0, x)=x$, and possesses the semigroup property $s(t, s(\tau, x))=s(t+\tau, x)$ for all $t, \tau \geq 0$ and $x \in \mathcal{D}$. Given $t \geq 0$ and $x \in \mathcal{D}$, we denote the map $s(t, \cdot): \mathcal{D} \rightarrow \mathcal{D}$ by $s_{t}$ and the map $s(\cdot, x):[0, \infty) \rightarrow \mathcal{D}$ by $s^{x}$. For every $t \in \mathbb{R}$, the map $s_{t}$ is a homeomorphism and has the inverse $s_{-t}$.

The orbit $\mathcal{O}_{x}$ of a point $x \in \mathcal{D}$ is the set $s^{x}([0, \infty))$. A set $\mathcal{D}_{\mathrm{c}} \subseteq \mathcal{D}$ is positively invariant relative to (1) if $s_{t}\left(\mathcal{D}_{\mathrm{c}}\right) \subseteq \mathcal{D}_{\mathrm{c}}$ for all $t \geq 0$ or, equivalently, $\mathcal{D}_{\mathrm{c}}$ contains the orbits of all its points. The set $\mathcal{D}_{\mathrm{C}}$ is invariant relative to (1) if $s_{t}\left(\mathcal{D}_{\mathrm{c}}\right)=\mathcal{D}_{\mathrm{c}}$ for all $t \geq 0$. The positive limit set of $x \in \mathbb{R}^{q}$ is the set $\omega(x)$ of all subsequential limits of sequences of the form $\left\{s\left(t_{i}, x\right)\right\}_{i=0}^{\infty}$, where $\left\{t_{i}\right\}_{i=0}^{\infty}$ is an increasing divergent sequence in $[0, \infty) . \omega(x)$ is closed and invariant, and $\overline{\mathcal{O}}_{x}=\mathcal{O}_{x} \cup$ $\omega(x)$ (Haddad \& Chellaboina (2008)). In addition, for every $x \in \mathbb{R}^{q}$ that has bounded positive orbits, $\omega(x)$ is nonempty and compact, and, for every neighborhood $\mathcal{N}$ of $\omega(x)$, there exists $T>0$ such that $s_{t}(x) \in \mathcal{N}$ for every $t>T$ (Haddad \& Chellaboina (2008)). Furthermore, $x_{\mathrm{e}} \in \mathcal{D}$ is an equilibrium point of (1) if and only if $f\left(x_{\mathrm{e}}\right)=0$ or, equivalently, $s\left(t, x_{\mathrm{e}}\right)=x_{\mathrm{e}}$ for all $t \geq 0$. Finally, recall that if all solutions to (1) are bounded, then it follows from the Peano-Cauchy theorem (Haddad \& Chellaboina, 2008, p. 76) that $\mathcal{I}_{x_{0}}=\mathbb{R}$.

Definition 21 (Haddad et al., 2010, pp. 9,10) Let $f=\left[f_{1}, \ldots, f_{n}\right]^{\mathrm{T}}: \mathcal{D} \subseteq \overline{\mathbb{R}}_{+}^{n} \rightarrow \mathbb{R}^{n}$. Then $f$ is essentially nonnegative if $f_{i}(x) \geq 0$, for all $i=1, \ldots, n$, and $x \in \overline{\mathbb{R}}_{+}^{n}$ such that $x_{i}=0$, where $x_{i}$ denotes the ith component of $x$. 
Proposition 21 (Haddad et al., 2010, p. 12) Suppose $\overline{\mathbb{R}}_{+}^{n} \subset \mathcal{D}$. Then $\overline{\mathbb{R}}_{+}^{n}$ is an invariant set with respect to (1) if and only if $f: \mathcal{D} \rightarrow \mathbb{R}^{n}$ is essentially nonnegative.

Definition 22 (Haddad et al., 2010, pp. 13,23) An equilibrium solution $x(t) \equiv x_{\mathrm{e}} \in \overline{\mathbb{R}}_{+}^{n}$ to (1) is Lyapunov stable with respect to $\overline{\mathbb{R}}_{+}^{n}$ if, for all $\varepsilon>0$, there exists $\delta=\delta(\varepsilon)>0$ such that if $x \in \mathcal{B}_{\delta}\left(x_{\mathrm{e}}\right) \cap \overline{\mathbb{R}}_{+}^{n}$, then $x(t) \in \mathcal{B}_{\varepsilon}\left(x_{\mathrm{e}}\right) \cap \overline{\mathbb{R}}_{+}^{n}, t \geq 0$. An equilibrium solution $x(t) \equiv x_{\mathrm{e}} \in \overline{\mathbb{R}}_{+}^{n}$ to (1) is semistable with respect to $\overline{\mathbb{R}}_{+}^{n}$ if it is Lyapunov stable with respect to $\overline{\mathbb{R}}_{+}^{n}$ and there exists $\delta>0$ such that if $x_{0} \in \mathcal{B}_{\delta}\left(x_{\mathrm{e}}\right) \cap \overline{\mathbb{R}}_{+}^{n}$, then $\lim _{t \rightarrow \infty} x(t)$ exists and corresponds to a Lyapunov stable equilibrium point with respect to $\overline{\mathbb{R}}_{+}^{n}$. The system (1) is said to be semistable with respect to $\overline{\mathbb{R}}_{+}^{n}$ if every equilibrium point of (1) is semistable with respect to $\overline{\mathbb{R}}_{+}^{n}$. The system (1) is said to be globally semistable with respect to $\overline{\mathbb{R}}_{+}^{n}$ if (1) is semistable with respect to $\overline{\mathbb{R}}_{+}^{n}$ and, for every $x_{0} \in \overline{\mathbb{R}}_{+}^{n}$, $\lim _{t \rightarrow \infty} x(t)$ exists.

Proposition 22 (Haddad et al., 2010, p. 22) Consider the nonlinear dynamical system (1) where $f$ is essentially nonnegative and let $x \in \overline{\mathbb{R}}_{+}^{n}$. If the positive limit set of (1) contains a Lyapunov stable (with respect to $\overline{\mathbb{R}}_{+}^{n}$ ) equilibrium point $y$, then $y=\lim _{t \rightarrow \infty} s(t, x)$.

\section{Interconnected thermodynamic systems: A state space energy flow perspective}

The fundamental and unifying concept in the analysis of thermodynamic systems is the concept of energy. The energy of a state of a dynamical system is the measure of its ability to produce changes (motion) in its own system state as well as changes in the system states of its surroundings. These changes occur as a direct consequence of the energy flow between different subsystems within the dynamical system. Heat (energy) is a fundamental concept of thermodynamics involving the capacity of hot bodies (more energetic subsystems) to produce work. As in thermodynamic systems, dynamical systems can exhibit energy (due to friction) that becomes unavailable to do useful work. This in turn contributes to an increase in system entropy, a measure of the tendency of a system to lose the ability to do useful work. In this section, we use the state space formalism to construct a mathematical model of a thermodynamic system that is consistent with basic thermodynamic principles.

Specifically, we consider a large-scale system model with a combination of subsystems (compartments or parts) that is perceived as a single entity. For each subsystem (compartment) making up the system, we postulate the existence of an energy state variable such that the knowledge of these subsystem state variables at any given time $t=t_{0}$, together with the knowledge of any inputs (heat fluxes) to each of the subsystems for time $t \geq t_{0}$, completely determines the behavior of the system for any given time $t \geq t_{0}$. Hence, the (energy) state of our dynamical system at time $t$ is uniquely determined by the state at time $t_{0}$ and any external inputs for time $t \geq t_{0}$ and is independent of the state and inputs before time $t_{0}$.

More precisely, we consider a large-scale interconnected dynamical system composed of a large number of units with aggregated (or lumped) energy variables representing homogenous groups of these units. If all the units comprising the system are identical (that is, the system is perfectly homogeneous), then the behavior of the dynamical system can be captured by that of a single plenipotentiary unit. Alternatively, if every interacting system unit is distinct, then the resulting model constitutes a microscopic system. To develop a middle-ground thermodynamic model placed between complete aggregation (classical thermodynamics) and complete disaggregation (statistical thermodynamics), we subdivide 




Fig. 1. Interconnected dynamical system $\mathcal{G}$.

the large-scale dynamical system into a finite number of compartments, each formed by a large number of homogeneous units. Each compartment represents the energy content of the different parts of the dynamical system, and different compartments interact by exchanging heat. Thus, our compartmental thermodynamic model utilizes subsystems or compartments to describe the energy distribution among distinct regions in space with intercompartmental flows representing the heat transfer between these regions. Decreasing the number of compartments results in a more aggregated or homogeneous model, whereas increasing the number of compartments leads to a higher degree of disaggregation resulting in a heterogeneous model.

To formulate our state space thermodynamic model, consider the interconnected dynamical system $\mathcal{G}$ shown in Figure 1 involving energy exchange between $q$ interconnected subsystems. Let $E_{i}:[0, \infty) \rightarrow \overline{\mathbb{R}}_{+}$denote the energy (and hence a nonnegative quantity) of the $i$ th subsystem, let $S_{i}:[0, \infty) \rightarrow \mathbb{R}$ denote the external power (heat flux) supplied to (or extracted from) the $i$ th subsystem, let $\phi_{i j}: \overline{\mathbb{R}}_{+}^{q} \rightarrow \mathbb{R}, i \neq j, i, j=1, \ldots, q$, denote the net instantaneous rate of energy (heat) flow from the $j$ th subsystem to the $i$ th subsystem, and let $\sigma_{i i}: \overline{\mathbb{R}}_{+}^{q} \rightarrow \overline{\mathbb{R}}_{+}, i=$ $1, \ldots, q$, denote the instantaneous rate of energy (heat) dissipation from the $i$ th subsystem to the environment. Here, we assume that $\phi_{i j}: \overline{\mathbb{R}}_{+}^{q} \rightarrow \mathbb{R}, i \neq j, i, j=1, \ldots, q$, and $\sigma_{i i}: \overline{\mathbb{R}}_{+}^{q} \rightarrow \overline{\mathbb{R}}_{+}$, $i=1, \ldots, q$, are locally Lipschitz continuous on $\overline{\mathbb{R}}_{+}^{q}$ and $S_{i}:[0, \infty) \rightarrow \mathbb{R}, i=1, \ldots, q$, are bounded piecewise continuous functions of time. 
An energy balance for the $i$ th subsystem yields

$$
E_{i}(T)=E_{i}\left(t_{0}\right)+\left[\sum_{j=1, j \neq i}^{q} \int_{t_{0}}^{T} \phi_{i j}(E(t)) \mathrm{d} t\right]-\int_{t_{0}}^{T} \sigma_{i i}(E(t)) \mathrm{d} t+\int_{t_{0}}^{T} S_{i}(t) \mathrm{d} t, \quad T \geq t_{0}
$$

or, equivalently, in vector form,

$$
E(T)=E\left(t_{0}\right)+\int_{t_{0}}^{T} w(E(t)) \mathrm{d} t-\int_{t_{0}}^{T} d(E(t)) \mathrm{d} t+\int_{t_{0}}^{T} S(t) \mathrm{d} t, \quad T \geq t_{0},
$$

where $E(t) \triangleq\left[E_{1}(t), \ldots, E_{q}(t)\right]^{\mathrm{T}}, t \geq t_{0}$, is the system energy state, $d(E(t)) \triangleq\left[\sigma_{11}(E(t)), \ldots\right.$, $\left.\sigma_{q q}(E(t))\right]^{\mathrm{T}}, t \geq t_{0}$, is the system dissipation, $S(t) \triangleq\left[S_{1}(t), \ldots, S_{q}(t)\right]^{\mathrm{T}}, t \geq t_{0}$, is the system heat flux, and $w=\left[w_{1}, \ldots, w_{q}\right]^{\mathrm{T}}: \overline{\mathbb{R}}_{+}^{q} \rightarrow \mathbb{R}^{q}$ is such that

$$
w_{i}(E)=\sum_{j=1, j \neq i}^{q} \phi_{i j}(E), \quad E \in \overline{\mathbb{R}}_{+}^{q} .
$$

Since $\phi_{i j}: \overline{\mathbb{R}}_{+}^{q} \rightarrow \mathbb{R}, i \neq j, i, j=1, \ldots, q$, denotes the net instantaneous rate of energy flow from the $j$ th subsystem to the $i$ th subsystem, it is clear that $\phi_{i j}(E)=-\phi_{j i}(E), E \in \overline{\mathbb{R}}_{+}^{q}, i \neq j, i, j=$ $1, \ldots, q$, which further implies that $\mathbf{e}^{\mathrm{T}} w(E)=0, E \in \overline{\mathbb{R}}_{+}^{q}$.

Note that (2) yields a conservation of energy equation and implies that the energy stored in the $i$ th subsystem is equal to the external energy supplied to (or extracted from) the $i$ th subsystem plus the energy gained by the $i$ th subsystem from all other subsystems due to subsystem coupling minus the energy dissipated from the $i$ th subsystem to the environment. Equivalently, (2) can be rewritten as

$$
\dot{E}_{i}(t)=\left[\sum_{j=1, j \neq i}^{q} \phi_{i j}(E(t))\right]-\sigma_{i i}(E(t))+S_{i}(t), \quad E_{i}\left(t_{0}\right)=E_{i 0}, \quad t \geq t_{0},
$$

or, in vector form,

$$
\dot{E}(t)=w(E(t))-d(E(t))+S(t), \quad E\left(t_{0}\right)=E_{0}, \quad t \geq t_{0},
$$

where $E_{0} \triangleq\left[E_{10}, \ldots, E_{q 0}\right]^{\mathrm{T}}$, yielding a power balance equation that characterizes energy flow between subsystems of the interconnected dynamical system $\mathcal{G}$. We assume that $\phi_{i j}(E) \geq$ $0, E \in \overline{\mathbb{R}}_{+}^{q}$, whenever $E_{i}=0, i \neq j, i, j=1, \ldots, q$, and $\sigma_{i i}(E)=0$, whenever $E_{i}=0, i=$ $1, \ldots, q$. The above constraint implies that if the energy of the $i$ th subsystem of $\mathcal{G}$ is zero, then this subsystem cannot supply any energy to its surroundings nor can it dissipate energy to the environment. In this case, $w(E)-d(E), E \in \overline{\mathbb{R}}_{+}^{q}$, is essentially nonnegative (Haddad \& Chellaboina (2005)). Thus, if $S(t) \equiv 0$, then, by Proposition 21, the solutions to (6) are nonnegative for all nonnegative initial conditions. See Haddad \& Chellaboina (2005); Haddad et al. $(2005 ; 2010)$ for further details.

Since our thermodynamic compartmental model involves intercompartmental flows representing energy transfer between compartments, we can use graph-theoretic notions with undirected graph topologies (i.e., bidirectional energy flows) to capture the compartmental system interconnections. Graph theory (Diestel (1997); Godsil \& Royle (2001)) can be useful 
in the analysis of the connectivity properties of compartmental systems. In particular, an undirected graph can be constructed to capture a compartmental model in which the compartments are represented by nodes and the flows are represented by edges or arcs. In this case, the environment must also be considered as an additional node.

For the interconnected dynamical system $\mathcal{G}$ with the power balance equation (6), we define a connectivity matrix ${ }^{1} \mathcal{C} \in \mathbb{R}^{q \times q}$ such that for $i \neq j, i, j=1, \ldots, q, \mathcal{C}_{(i, j)} \triangleq 1$ if $\phi_{i j}(E) \not \equiv 0$ and $\mathcal{C}_{(i, j)} \triangleq 0$ otherwise, and $\mathcal{C}_{(i, i)} \triangleq-\sum_{k=1, k \neq i}^{q} \mathcal{C}_{(k, i)}, i=1, \ldots, q$. Recall that if $\operatorname{rank} \mathcal{C}=q-1$, then $\mathcal{G}$ is strongly connected (Haddad et al. (2005)) and energy exchange is possible between any two subsystems of $\mathcal{G}$. The next definition introduces a notion of entropy for the interconnected dynamical system $\mathcal{G}$.

Definition 3.1 Consider the interconnected dynamical system $\mathcal{G}$ with the power balance equation (6). A continuously differentiable, strictly concave function $\mathcal{S}: \overline{\mathbb{R}}_{+}^{q} \rightarrow \mathbb{R}$ is called the entropy function of $\mathcal{G}$ if

$$
\left(\frac{\partial \mathcal{S}(E)}{\partial E_{i}}-\frac{\partial \mathcal{S}(E)}{\partial E_{j}}\right) \phi_{i j}(E) \geq 0, \quad E \in \overline{\mathbb{R}}_{+,}^{q} \quad i \neq j, \quad i, j=1, \ldots, q,
$$

and $\frac{\partial \mathcal{S}(E)}{\partial E_{i}}=\frac{\partial \mathcal{S}(E)}{\partial E_{j}}$ if and only if $\phi_{i j}(E)=0$ with $\mathcal{C}_{(i, j)}=1, i \neq j, i, j=1, \ldots, q$.

It follows from Definition 3.1 that for an isolated system $\mathcal{G}$, that is, $S(t) \equiv 0$ and $d(E) \equiv 0$, the entropy function of $\mathcal{G}$ is a nondecreasing function of time. To see this, note that

$$
\begin{aligned}
\dot{\mathcal{S}}(E) & =\frac{\partial \mathcal{S}(E)}{\partial E} \dot{E} \\
& =\sum_{i=1}^{q} \frac{\partial \mathcal{S}(E)}{\partial E_{i}} \sum_{j=1, j \neq i}^{q} \phi_{i j}(E) \\
& =\sum_{i=1}^{q} \sum_{j=i+1}^{q}\left(\frac{\partial \mathcal{S}(E)}{\partial E_{i}}-\frac{\partial \mathcal{S}(E)}{\partial E_{j}}\right) \phi_{i j}(E) \\
& \geq 0, \quad E \in \overline{\mathbb{R}}_{+}^{q},
\end{aligned}
$$

where $\frac{\partial \mathcal{S}(E)}{\partial E} \triangleq\left[\frac{\partial \mathcal{S}(E)}{\partial E_{1}}, \ldots, \frac{\partial \mathcal{S}(E)}{\partial E_{q}}\right]$ and where we used the fact that $\phi_{i j}(E)=-\phi_{j i}(E), E \in \overline{\mathbb{R}}_{+}^{q}$, $i \neq j, i, j=1, \ldots, q$.

Proposition 3.1 Consider the isolated (i.e., $S(t) \equiv 0$ and $d(E) \equiv 0$ ) interconnected dynamical system $\mathcal{G}$ with the power balance equation (6). Assume that rankC $=q-1$ and there exists an entropy function $\mathcal{S}: \overline{\mathbb{R}}_{+}^{q} \rightarrow \mathbb{R}$ of $\mathcal{G}$. Then, $\sum_{j=1}^{q} \phi_{i j}(E)=0$ for all $i=1, \ldots, q$ if and only if $\frac{\partial \mathcal{S}(E)}{\partial E_{1}}=\cdots=\frac{\partial \mathcal{S}(E)}{\partial E_{q}}$. Furthermore, the set of nonnegative equilibrium states of (6) is given by $\mathcal{E}_{0} \triangleq\left\{E \in \overline{\mathbb{R}}_{+}^{q}: \frac{\partial \mathcal{S}(E)}{\partial E_{1}}=\cdots=\frac{\partial \mathcal{S}(E)}{\partial E_{q}}\right\}$.

\footnotetext{
${ }^{1}$ The negative of the connectivity matrix, that is, $-\mathcal{C}$, is known as the graph Laplacian in the literature.
} 
Proof. If $\frac{\partial \mathcal{S}(E)}{\partial E_{i}}=\frac{\partial \mathcal{S}(E)}{\partial E_{j}}$, then $\phi_{i j}(E)=0$ for all $i, j=1, \ldots, q$, which implies that $\sum_{j=1}^{q} \phi_{i j}(E)=0$ for all $i=1, \ldots, q$. Conversely, assume that $\sum_{j=1}^{q} \phi_{i j}(E)=0$ for all $i=1, \ldots, q$, and, since $\mathcal{S}$ is an entropy function of $\mathcal{G}$, it follows that

$$
\begin{aligned}
0 & =\sum_{i=1}^{q} \sum_{j=1}^{q} \frac{\partial \mathcal{S}(E)}{\partial E_{i}} \phi_{i j}(E) \\
& =\sum_{i=1}^{q-1} \sum_{j=i+1}^{q}\left(\frac{\partial \mathcal{S}(E)}{\partial E_{i}}-\frac{\partial \mathcal{S}(E)}{\partial E_{j}}\right) \phi_{i j}(E) \\
& \geq 0
\end{aligned}
$$

where we have used the fact that $\phi_{i j}(E)=-\phi_{j i}(E)$ for all $i, j=1, \ldots, q$. Hence,

$$
\left(\frac{\partial \mathcal{S}(E)}{\partial E_{i}}-\frac{\partial \mathcal{S}(E)}{\partial E_{j}}\right) \phi_{i j}(E)=0
$$

for all $i, j=1, \ldots, q$. Now, the result follows from the fact that rank $\mathcal{C}=q-1$.

Theorem 3.1 Consider the isolated (i.e., $S(t) \equiv 0$ and $d(E) \equiv 0$ ) interconnected dynamical system $\mathcal{G}$ with the power balance equation (6). Assume that rankC $=q-1$ and there exists an entropy function $\mathcal{S}: \overline{\mathbb{R}}_{+}^{q} \rightarrow \mathbb{R}$ of $\mathcal{G}$. Then the isolated system $\mathcal{G}$ is globally semistable with respect to $\overline{\mathbb{R}}_{+}^{q}$.

Proof. Since $w(\cdot)$ is essentially nonnegative, it follows from Proposition 21 that $E(t) \in \overline{\mathbb{R}}_{+}^{q}$, $t \geq t_{0}$, for all $E_{0} \in \overline{\mathbb{R}}_{+}^{q}$. Furthermore, note that since $\mathbf{e}^{\mathrm{T}} w(E)=0, E \in \overline{\mathbb{R}}_{+}^{q}$, it follows that $\mathbf{e}^{\mathrm{T}} \dot{E}(t)=0, t \geq t_{0}$. In this case, $\mathbf{e}^{\mathrm{T}} E(t)=\mathbf{e}^{\mathrm{T}} E_{0}, t \geq t_{0}$, which implies that $E(t), t \geq t_{0}$, is bounded for all $E_{0} \in \overline{\mathbb{R}}_{+}^{q}$. Now, it follows from (8) that $\mathcal{S}(E(t)), t \geq t_{0}$, is a nondecreasing function of time, and hence, by the Krasovskii-LaSalle theorem (Haddad \& Chellaboina (2008)), $E(t) \rightarrow$ $\mathcal{R} \triangleq\left\{E \in \overline{\mathbb{R}}_{+}^{q}: \dot{\mathcal{S}}(E)=0\right\}$ as $t \rightarrow \infty$. Next, it follows from (8), Definition 3.1, and the fact that $\operatorname{rank} \mathcal{C}=q-1$, that $\mathcal{R}=\left\{E \in \overline{\mathbb{R}}_{+}^{q}: \frac{\partial \mathcal{S}(E)}{\partial E_{1}}=\cdots=\frac{\partial \mathcal{S}(E)}{\partial E_{q}}\right\}=\mathcal{E}_{0}$.

Now, let $E_{\mathrm{e}} \in \mathcal{E}_{0}$ and consider the continuously differentiable function $V: \mathbb{R}^{q} \rightarrow \mathbb{R}$ defined by

$$
V(E) \triangleq \mathcal{S}\left(E_{\mathrm{e}}\right)-\mathcal{S}(E)-\lambda_{\mathrm{e}}\left(\mathbf{e}^{\mathrm{T}} E_{\mathrm{e}}-\mathbf{e}^{\mathrm{T}} E\right),
$$

where $\lambda_{\mathrm{e}} \triangleq \frac{\partial \mathcal{S}}{\partial E_{1}}\left(E_{\mathrm{e}}\right)$. Next, note that $V\left(E_{\mathrm{e}}\right)=0, \frac{\partial V}{\partial E}\left(E_{\mathrm{e}}\right)=-\frac{\partial \mathcal{S}}{\partial E}\left(E_{\mathrm{e}}\right)+\lambda_{\mathrm{e}} \mathbf{e}^{\mathrm{T}}=0$, and, since $\mathcal{S}(\cdot)$ is a strictly concave function, $\frac{\partial^{2} V}{\partial E^{2}}\left(E_{\mathrm{e}}\right)=-\frac{\partial^{2} S}{\partial E^{2}}\left(E_{\mathrm{e}}\right)>0$, which implies that $V(\cdot)$ admits a local minimum at $E_{\mathrm{e}}$. Thus, $V\left(E_{\mathrm{e}}\right)=0$, there exists $\delta>0$ such that $V(E)>0, E \in \mathcal{B}_{\delta}\left(E_{\mathrm{e}}\right) \backslash\left\{E_{\mathrm{e}}\right\}$, and $\dot{V}(E)=-\dot{\mathcal{S}}(E) \leq 0$ for all $E \in \mathcal{B}_{\delta}\left(E_{\mathrm{e}}\right) \backslash\left\{E_{\mathrm{e}}\right\}$, which shows that $V(\cdot)$ is a Lyapunov function for $\mathcal{G}$ and $E_{\mathrm{e}}$ is a Lyapunov stable equilibrium of $\mathcal{G}$. Finally, since, for every $E_{0} \in \overline{\mathbb{R}}_{+}^{n}, E(t) \rightarrow \mathcal{E}_{0}$ as $t \rightarrow \infty$ and every equilibrium point of $\mathcal{G}$ is Lyapunov stable, it follows from Proposition 22 that $\mathcal{G}$ is globally semistable with respect to $\overline{\mathbb{R}}_{+}^{q}$.

In classical thermodynamics, the partial derivative of the system entropy with respect to the system energy defines the reciprocal of the system temperature. Thus, for the interconnected dynamical system $\mathcal{G}$,

$$
T_{i} \triangleq\left(\frac{\partial \mathcal{S}(E)}{\partial E_{i}}\right)^{-1}, \quad i=1, \ldots, q,
$$


represents the temperature of the $i$ th subsystem. Condition (7) is a manifestation of the second law of thermodynamics and implies that if the temperature of the $j$ th subsystem is greater than the temperature of the $i$ th subsystem, then energy (heat) flows from the $j$ th subsystem to the $i$ th subsystem. Furthermore, $\frac{\partial \mathcal{S}(E)}{\partial E_{i}}=\frac{\partial \mathcal{S}(E)}{\partial E_{j}}$ if and only if $\phi_{i j}(E)=0$ with $\mathcal{C}_{(i, j)}=1, i \neq j, i, j=$ $1, \ldots, q$, implies that temperature equality is a necessary and sufficient condition for thermal equilibrium. This is a statement of the zeroth law of thermodynamics. As a result, Theorem 3.1 shows that, for a strongly connected system $\mathcal{G}$, the subsystem energies converge to the set of equilibrium states where the temperatures of all subsystems are equal. This phenomenon is known as equipartition of temperature (Haddad et al. (2010)) and is an emergent behavior in thermodynamic systems. In particular, all the system energy is eventually transferred into heat at a uniform temperature, and hence, all dynamical processes in $\mathcal{G}$ (system motions) would cease.

The following result presents a sufficient condition for energy equipartition of the system, that is, the energies of all subsystems are equal. And this state of energy equipartition is uniquely determined by the initial energy in the system.

Theorem 3.2 Consider the isolated (i.e., $S(t) \equiv 0$ and $d(E) \equiv 0$ ) interconnected dynamical system $\mathcal{G}$ with the power balance equation (6). Assume that rankC $=q-1$ and there exists a continuously differentiable, strictly concave function $f: \overline{\mathbb{R}}_{+} \rightarrow \mathbb{R}$ such that the entropy function $\mathcal{S}: \overline{\mathbb{R}}_{+}^{q} \rightarrow \mathbb{R}$ of $\mathcal{G}$ is given by $\mathcal{S}(E)=\sum_{i=1}^{q} f\left(E_{i}\right)$. Then, the set of nonnegative equilibrium states of (6) is given by $\mathcal{E}_{0}=\{\alpha \mathbf{e}: \alpha \geq 0\}$ and $\mathcal{G}$ is semistable with respect to $\overline{\mathbb{R}}_{+}^{q}$. Furthermore, $E(t) \rightarrow \frac{1}{q} \mathbf{e e}^{\mathrm{T}} E\left(t_{0}\right)$ as $t \rightarrow \infty$ and $\frac{1}{q} \mathbf{e e}^{\mathrm{T}} E\left(t_{0}\right)$ is a semistable equilibrium state of $\mathcal{G}$.

Proof. First, note that since $f(\cdot)$ is a continuously differentiable, strictly concave function it follows that

$$
\left(\frac{\mathrm{d} f}{\mathrm{~d} E_{i}}-\frac{\mathrm{d} f}{\mathrm{~d} E_{j}}\right)\left(E_{i}-E_{j}\right) \leq 0, \quad E \in \overline{\mathbb{R}}_{+,}^{q} \quad i, j=1, \ldots, q,
$$

which implies that (7) is equivalent to

$$
\left(E_{i}-E_{j}\right) \phi_{i j}(E) \leq 0, \quad E \in \overline{\mathbb{R}}_{+}^{q}, \quad i \neq j, \quad i, j=1, \ldots, q,
$$

and $E_{i}=E_{j}$ if and only if $\phi_{i j}(E)=0$ with $\mathcal{C}_{(i, j)}=1, i \neq j, i, j=1, \ldots, q$. Hence, $-E^{\mathrm{T}} E$ is an entropy function of $\mathcal{G}$. Next, with $\mathcal{S}(E)=-\frac{1}{2} E^{\mathrm{T}} E$, it follows from Proposition 3.1 that $\mathcal{E}_{0}=\left\{\alpha \mathbf{e} \in \overline{\mathbb{R}}_{+}^{q}, \alpha \geq 0\right\}$. Now, it follows from Theorem 3.1 that $\mathcal{G}$ is globally semistable with respect to $\overline{\mathbb{R}}_{+}^{q}$. Finally, since $\mathbf{e}^{\mathrm{T}} E(t)=\mathbf{e}^{\mathrm{T}} E\left(t_{0}\right)$ and $E(t) \rightarrow \mathcal{M}$ as $t \rightarrow \infty$, it follows that $E(t) \rightarrow \frac{1}{q} \mathbf{e e}^{\mathrm{T}} E\left(t_{0}\right)$ as $t \rightarrow \infty$. Hence, with $\alpha=\frac{1}{q} \mathbf{e}^{\mathrm{T}} E\left(t_{0}\right), \alpha \mathbf{e}=\frac{1}{q} \mathbf{e e}^{\mathrm{T}} E\left(t_{0}\right)$ is a semistable equilibrium state of (6).

If $f\left(E_{i}\right)=\log _{e}\left(c+E_{i}\right)$, where $c>0$, so that $\mathcal{S}(E)=\sum_{i=1}^{q} \log _{e}\left(c+E_{i}\right)$, then it follows from Theorem 3.2 that $\mathcal{E}_{0}=\{\alpha \mathbf{e}: \alpha \geq 0\}$ and the isolated (i.e., $S(t) \equiv 0$ and $d(E) \equiv 0$ ) interconnected dynamical system $\mathcal{G}$ with the power balance equation (6) is semistable. In this case, the absolute temperature of the $i$ th compartment is given by $c+E_{i}$. Similarly, if $\mathcal{S}(E)=-\frac{1}{2} E^{\mathrm{T}} E$, then it follows from Theorem 3.2 that $\mathcal{E}_{0}=\{\alpha \mathbf{e}: \alpha \geq 0\}$ and the isolated (i.e., $S(t) \equiv 0$ and $d(E) \equiv 0$ ) interconnected dynamical system $\mathcal{G}$ with the power balance equation (6) is semistable. In both these cases, $E(t) \rightarrow \frac{1}{q} \mathbf{e e}^{\mathrm{T}} E\left(t_{0}\right)$ as $t \rightarrow \infty$. This shows that the steady-state energy of the isolated interconnected dynamical system $\mathcal{G}$ is given by 
$\frac{1}{q} \mathbf{e e}^{\mathrm{T}} E\left(t_{0}\right)=\frac{1}{q} \sum_{i=1}^{q} E_{i}\left(t_{0}\right) \mathbf{e}$, and hence, is uniformly distributed over all subsystems of $\mathcal{G}$. This phenomenon is known as energy equipartition (Haddad et al. (2005)). The aforementioned forms of $\mathcal{S}(E)$ were extensively discussed in the recent book by Haddad et al. (2005) where $\mathcal{S}(E)=\sum_{i=1}^{q} \log _{e}\left(c+E_{i}\right)$ and $-\mathcal{S}(E)=\frac{1}{2} E^{\mathrm{T}} E$ are referred to, respectively, as the entropy and the ectropy functions of the interconnected dynamical system $\mathcal{G}$.

\section{Work energy, free energy, heat flow, and Clausius' inequality}

In this section, we augment our thermodynamic energy flow model $\mathcal{G}$ with an additional (deformation) state representing subsystem volumes in order to introduce the notion of work into our thermodynamically consistent state space energy flow model. Specifically, we assume that each subsystem can perform (positive) work on the environment as well as the environment can perform (negative) work on the subsystems. The rate of work done by the $i$ th subsystem on the environment is denoted by $d_{\mathrm{w} i}: \overline{\mathbb{R}}_{+}^{q} \times \mathbb{R}_{+}^{q} \rightarrow \overline{\mathbb{R}}_{+}, i=1, \ldots, q$, the rate of work done by the environment on the $i$ th subsystem is denoted by $S_{\mathrm{w} i}:[0, \infty) \rightarrow \overline{\mathbb{R}}_{+}, i=1, \ldots, q$, and the volume of the $i$ th subsystem is denoted by $V_{i}:[0, \infty) \rightarrow \mathbb{R}_{+}, i=1, \ldots, q$. The net work done by each subsystem on the environment satisfies

$$
p_{i}(E, V) \mathrm{d} V_{i}=\left(d_{\mathrm{w} i}(E, V)-S_{\mathrm{w} i}(t)\right) \mathrm{d} t,
$$

where $p_{i}(E, V), i=1, \ldots, q$, denotes the pressure in the $i$ th subsystem and $V \triangleq\left[V_{1}, \ldots, V_{q}\right]^{\mathrm{T}}$.

Furthermore, in the presence of work, the energy balance equation (5) for each subsystem can be rewritten as

$$
\mathrm{d} E_{i}=w_{i}(E, V) \mathrm{d} t-\left(d_{\mathrm{w} i}(E, V)-S_{\mathrm{w} i}(t)\right) \mathrm{d} t-\sigma_{i i}(E, V) \mathrm{d} t+S_{i}(t) \mathrm{d} t,
$$

where $w_{i}(E, V) \triangleq \sum_{j=1, j \neq i}^{q} \phi_{i j}(E, V), \phi_{i j}: \overline{\mathbb{R}}_{+}^{q} \times \mathbb{R}_{+}^{q} \rightarrow \mathbb{R}, i \neq j, i, j=1, \ldots, q$, denotes the net instantaneous rate of energy (heat) flow from the $j$ th subsystem to the $i$ th subsystem, $\sigma_{i i}$ : $\overline{\mathbb{R}}_{+}^{q} \times \mathbb{R}_{+}^{q} \rightarrow \overline{\mathbb{R}}_{+}, i=1, \ldots, q$, denotes the instantaneous rate of energy dissipation from the $i$ th subsystem to the environment, and, as in Section $3, S_{i}:[0, \infty) \rightarrow \mathbb{R}, i=1, \ldots, q$, denotes the external power supplied to (or extracted from) the $i$ th subsystem. It follows from (10) and (11) that positive work done by a subsystem on the environment leads to a decrease in internal energy of the subsystem and an increase in the subsystem volume, which is consistent with the first law of thermodynamics.

The definition of entropy for $\mathcal{G}$ in the presence of work remains the same as in Definition 3.1 with $\mathcal{S}(E)$ replaced by $\mathcal{S}(E, V)$ and with all other conditions in the definition holding for every $V>>0$. Next, consider the $i$ th subsystem of $\mathcal{G}$ and assume that $E_{j}$ and $V_{j}, j \neq i, i=1, \ldots, q$, are constant. In this case, note that

$$
\frac{\mathrm{d} \mathcal{S}}{\mathrm{d} t}=\frac{\partial \mathcal{S}}{\partial E_{i}} \frac{\mathrm{d} E_{i}}{\mathrm{~d} t}+\frac{\partial \mathcal{S}}{\partial V_{i}} \frac{\mathrm{d} V_{i}}{\mathrm{~d} t}
$$

and define

$$
p_{i}(E, V) \triangleq\left(\frac{\partial \mathcal{S}}{\partial E_{i}}\right)^{-1}\left(\frac{\partial \mathcal{S}}{\partial V_{i}}\right), \quad i=1, \ldots, q
$$


It follows from (10) and (11) that, in the presence of work energy, the power balance equation (6) takes the new form involving energy and deformation states

$$
\begin{array}{r}
\dot{E}(t)=w(E(t), V(t))-d_{\mathrm{W}}(E(t), V(t))+S_{\mathrm{W}}(t)-d(E(t), V(t))+S(t), \\
E\left(t_{0}\right)=E_{0}, \quad t \geq t_{0}, \\
\dot{V}(t)=D(E(t), V(t))\left(d_{\mathrm{W}}(E(t), V(t))-S_{\mathrm{W}}(t)\right), \quad V\left(t_{0}\right)=V_{0},
\end{array}
$$

where $w(E, V) \triangleq\left[w_{1}(E, V), \ldots, w_{q}(E, V)\right]^{\mathrm{T}}, d_{\mathrm{w}}(E, V) \triangleq\left[d_{\mathrm{w} 1}(E, V), \ldots, d_{\mathrm{w} q}(E, V)\right]^{\mathrm{T}}, S_{\mathrm{w}}(t) \triangleq$ $\left[S_{\mathrm{w} 1}(t), \ldots, S_{\mathrm{w} q}(t)\right]^{\mathrm{T}}, d(E, V) \triangleq\left[\sigma_{11}(E, V), \ldots, \sigma_{q q}(E, V)\right]^{\mathrm{T}}, S(t) \triangleq\left[S_{1}(t), \ldots, S_{q}(t)\right]^{\mathrm{T}}$, and

$$
D(E, V) \triangleq \operatorname{diag}\left[\left(\frac{\partial \mathcal{S}}{\partial E_{1}}\right)\left(\frac{\partial \mathcal{S}}{\partial V_{1}}\right)^{-1}, \ldots,\left(\frac{\partial \mathcal{S}}{\partial E_{q}}\right)\left(\frac{\partial \mathcal{S}}{\partial V_{q}}\right)^{-1}\right] .
$$

Note that

$$
\left(\frac{\partial \mathcal{S}(E, V)}{\partial V}\right) D(E, V)=\frac{\partial \mathcal{S}(E, V)}{\partial E} .
$$

The power balance and deformation equations (14) and (15) represent a statement of the first law of thermodynamics. To see this, define the work $L$ done by the interconnected dynamical system $\mathcal{G}$ over the time interval $\left[t_{1}, t_{2}\right]$ by

$$
L \triangleq \int_{t_{1}}^{t_{2}} \mathbf{e}^{\mathrm{T}}\left[d_{\mathrm{W}}(E(t), V(t))-S_{\mathrm{W}}(t)\right] \mathrm{d} t
$$

where $\left[E^{\mathrm{T}}(t), V^{\mathrm{T}}(t)\right]^{\mathrm{T}}, t \geq t_{0}$, is the solution to (14) and (15). Now, premultiplying (14) by $\mathbf{e}^{\mathrm{T}}$ and using the fact that $\mathbf{e}^{\overline{\mathrm{T}}} w(E, V)=0$, it follows that

$$
\Delta U=-L+Q,
$$

where $\Delta U=U\left(t_{2}\right)-U\left(t_{1}\right) \triangleq \mathbf{e}^{\mathrm{T}} E\left(t_{2}\right)-\mathbf{e}^{\mathrm{T}} E\left(t_{1}\right)$ denotes the variation in the total energy of the interconnected system $\mathcal{G}$ over the time interval $\left[t_{1}, t_{2}\right]$ and

$$
Q \triangleq \int_{t_{1}}^{t_{2}} \mathbf{e}^{\mathrm{T}}[S(t)-d(E(t), V(t))] \mathrm{d} t
$$

denotes the net energy received by $\mathcal{G}$ in forms other than work.

This is a statement of the first law of thermodynamics for the interconnected dynamical system $\mathcal{G}$ and gives a precise formulation of the equivalence between work and heat. This establishes that heat and mechanical work are two different aspects of energy. Finally, note that (15) is consistent with the classical thermodynamic equation for the rate of work done by the system $\mathcal{G}$ on the environment. To see this, note that (15) can be equivalently written as $\mathrm{d} L=\mathbf{e}^{\mathrm{T}} D^{-1}(E, V) \mathrm{d} V$, which, for a single subsystem with volume $V$ and pressure $p$, has the classical form

$$
\mathrm{d} L=p \mathrm{~d} V
$$


It follows from Definition 3.1 and (14)-(17) that the time derivative of the entropy function satisfies

$$
\begin{aligned}
\dot{\mathcal{S}}(E, V)= & \frac{\partial \mathcal{S}(E, V)}{\partial E} \dot{E}+\frac{\partial \mathcal{S}(E, V)}{\partial V} \dot{V} \\
= & \frac{\partial \mathcal{S}(E, V)}{\partial E} w(E, V)-\frac{\partial \mathcal{S}(E, V)}{\partial E}\left(d_{\mathrm{w}}(E, V)-S_{\mathrm{w}}(t)\right) \\
& -\frac{\partial \mathcal{S}(E, V)}{\partial E}(d(E, V)-S(t))+\frac{\partial \mathcal{S}(E, V)}{\partial V} D(E, V)\left(d_{\mathrm{w}}(E, V)-S_{\mathrm{w}}(t)\right) \\
= & \sum_{i=1}^{q} \frac{\partial \mathcal{S}(E, V)}{\partial E_{i}} \sum_{j=1, j \neq i}^{q} \phi_{i j}(E, V)+\sum_{i=1}^{q} \frac{\partial \mathcal{S}(E, V)}{\partial E_{i}}\left(S_{i}(t)-d_{i}(E, V)\right) \\
= & \sum_{i=1}^{q} \sum_{j=i+1}^{q}\left(\frac{\partial \mathcal{S}(E, V)}{\partial E_{i}}-\frac{\partial \mathcal{S}(E, V)}{\partial E_{j}}\right) \phi_{i j}(E, V) \\
& +\sum_{i=1}^{q} \frac{\partial \mathcal{S}(E, V)}{\partial E_{i}}\left(S_{i}(t)-d_{i}(E, V)\right) \\
\geq & \sum_{i=1}^{q} \frac{\partial \mathcal{S}(E, V)}{\partial E_{i}}\left(S_{i}(t)-d_{i}(E, V)\right), \quad(E, V) \in \overline{\mathbb{R}}_{+}^{q} \times \mathbb{R}_{+}^{q} .
\end{aligned}
$$

Noting that $\mathrm{d} Q_{i} \triangleq\left[S_{i}-\sigma_{i i}(E)\right] \mathrm{d} t, i=1, \ldots, q$, is the infinitesimal amount of the net heat received or dissipated by the $i$ th subsystem of $\mathcal{G}$ over the infinitesimal time interval $\mathrm{d} t$, it follows from (22) that

$$
\mathrm{d} \mathcal{S}(E) \geq \sum_{i=1}^{q} \frac{\mathrm{d} Q_{i}}{T_{i}} .
$$

Inequality (23) is the classical Clausius inequality for the variation of entropy during an infinitesimal irreversible transformation.

Note that for an adiabatically isolated interconnected dynamical system (i.e., no heat exchange with the environment), (22) yields the universal inequality

$$
\mathcal{S}\left(E\left(t_{2}\right), V\left(t_{2}\right)\right) \geq \mathcal{S}\left(E\left(t_{1}\right), V\left(t_{1}\right)\right), \quad t_{2} \geq t_{1},
$$

which implies that, for any dynamical change in an adiabatically isolated interconnected system $\mathcal{G}$, the entropy of the final system state can never be less than the entropy of the initial system state. In addition, in the case where $(E(t), V(t)) \in \mathcal{M}_{\mathrm{e}}, t \geq t_{0}$, where $\mathcal{M}_{\mathrm{e}} \triangleq\{(E, V) \in$ $\left.\overline{\mathbb{R}}_{+}^{q} \times \overline{\mathbb{R}}_{+}^{q}: E=\alpha \mathbf{e}, \alpha \geq 0, V \in \mathbb{R}_{+}^{q}\right\}$, it follows from Definition 3.1 and (22) that inequality (24) is satisfied as a strict inequality for all $(E, V) \in\left(\overline{\mathbb{R}}_{+}^{q} \times \overline{\mathbb{R}}_{+}^{q}\right) \backslash \mathcal{M}_{\mathrm{e}}$. Hence, it follows from Theorem 2.15 of Haddad et al. (2005) that the adiabatically isolated interconnected system $\mathcal{G}$ does not exhibit Poincaré recurrence in $\left(\overline{\mathbb{R}}_{+}^{q} \times \overline{\mathbb{R}}_{+}^{q}\right) \backslash \mathcal{M}$.

Next, we define the Gibbs free energy, the Helmholtz free energy, and the enthalpy functions for the interconnected dynamical system $\mathcal{G}$. For this exposition, we assume that the entropy of $\mathcal{G}$ is a sum of individual entropies of subsystems of $\mathcal{G}$, that is, $\mathcal{S}(E, V)=\sum_{i=1}^{q} \mathcal{S}_{i}\left(E_{i}, V_{i}\right),(E, V) \in$ 
$\overline{\mathbb{R}}_{+}^{q} \times \mathbb{R}_{+}^{q}$. In this case, the Gibbs free energy of $\mathcal{G}$ is defined by

$$
\begin{array}{r}
G(E, V) \triangleq \mathbf{e}^{\mathrm{T}} E-\sum_{i=1}^{q}\left(\frac{\partial \mathcal{S}(E, V)}{\partial E_{i}}\right)^{-1} \mathcal{S}_{i}\left(E_{i}, V_{i}\right)+\sum_{i=1}^{q}\left(\frac{\partial \mathcal{S}(E, V)}{\partial E_{i}}\right)^{-1}\left(\frac{\partial \mathcal{S}(E, V)}{\partial V_{i}}\right) V_{i} \\
(E, V) \in \overline{\mathbb{R}}_{+}^{q} \times \mathbb{R}_{+}^{q}
\end{array}
$$

the Helmholtz free energy of $\mathcal{G}$ is defined by

$$
F(E, V) \triangleq \mathbf{e}^{\mathrm{T}} E-\sum_{i=1}^{q}\left(\frac{\partial \mathcal{S}(E, V)}{\partial E_{i}}\right)^{-1} \mathcal{S}_{i}\left(E_{i}, V_{i}\right), \quad(E, V) \in \overline{\mathbb{R}}_{+}^{q} \times \mathbb{R}_{+}^{q},
$$

and the enthalpy of $\mathcal{G}$ is defined by

$$
H(E, V) \triangleq \mathbf{e}^{\mathrm{T}} E+\sum_{i=1}^{q}\left(\frac{\partial \mathcal{S}(E, V)}{\partial E_{i}}\right)^{-1}\left(\frac{\partial \mathcal{S}(E, V)}{\partial V_{i}}\right) V_{i}, \quad(E, V) \in \overline{\mathbb{R}}_{+}^{q} \times \mathbb{R}_{+}^{q} .
$$

Note that the above definitions for the Gibbs free energy, Helmholtz free energy, and enthalpy are consistent with the classical thermodynamic definitions given by $G(E, V)=U+p V-$ TS, $F(E, V)=U-T S$, and $H(E, V)=U+p V$, respectively. Furthermore, note that if the interconnected system $\mathcal{G}$ is isothermal and isobaric, that is, the temperatures of subsystems of $\mathcal{G}$ are equal and remain constant with

$$
\left(\frac{\partial \mathcal{S}(E, V)}{\partial E_{1}}\right)^{-1}=\cdots=\left(\frac{\partial \mathcal{S}(E, V)}{\partial E_{q}}\right)^{-1}=T>0
$$

and the pressure $p_{i}(E, V)$ in each subsystem of $\mathcal{G}$ remains constant, respectively, then any transformation in $\mathcal{G}$ is reversible.

The time derivative of $G(E, V)$ along the trajectories of (14) and (15) is given by

$$
\begin{aligned}
\dot{G}(E, V)= & \mathbf{e}^{\mathrm{T}} \dot{E}-\sum_{i=1}^{q}\left(\frac{\partial \mathcal{S}(E, V)}{\partial E_{i}}\right)^{-1}\left[\frac{\partial \mathcal{S}(E, V)}{\partial E_{i}} \dot{E}_{i}+\frac{\partial \mathcal{S}(E, V)}{\partial V_{i}} \dot{V}_{i}\right] \\
& +\sum_{i=1}^{q}\left(\frac{\partial \mathcal{S}(E, V)}{\partial E_{i}}\right)^{-1}\left(\frac{\partial \mathcal{S}(E, V)}{\partial V_{i}}\right) \dot{V}_{i} \\
= & 0,
\end{aligned}
$$

which is consistent with classical thermodynamics in the absence of chemical reactions.

For an isothermal interconnected dynamical system $\mathcal{G}$, the time derivative of $F(E, V)$ along the trajectories of (14) and (15) is given by

$$
\begin{aligned}
\dot{F}(E, V) & =\mathbf{e}^{\mathrm{T}} \dot{E}-\sum_{i=1}^{q}\left(\frac{\partial \mathcal{S}(E, V)}{\partial E_{i}}\right)^{-1}\left[\frac{\partial \mathcal{S}(E, V)}{\partial E_{i}} \dot{E}_{i}+\frac{\partial \mathcal{S}(E, V)}{\partial V_{i}} \dot{V}_{i}\right] \\
& =-\sum_{i=1}^{q}\left(\frac{\partial \mathcal{S}(E, V)}{\partial E_{i}}\right)^{-1}\left(\frac{\partial \mathcal{S}(E, V)}{\partial V_{i}}\right) \dot{V}_{i} \\
& =-\sum_{i=1}^{q}\left(d_{\mathrm{w} i}(E, V)-S_{\mathrm{w} i}(t)\right) \\
& =-L
\end{aligned}
$$


where $L$ is the net amount of work done by the subsystems of $\mathcal{G}$ on the environment. Furthermore, note that if, in addition, the interconnected system $\mathcal{G}$ is isochoric, that is, the volumes of each of the subsystems of $\mathcal{G}$ remain constant, then $\dot{F}(E, V)=0$. As we see in the next section, in the presence of chemical reactions the interconnected system $\mathcal{G}$ evolves such that the Helmholtz free energy is minimized.

Finally, for the isolated $(S(t) \equiv 0$ and $d(E, V) \equiv 0)$ interconnected dynamical system $\mathcal{G}$, the time derivative of $H(E, V)$ along the trajectories of (14) and (15) is given by

$$
\begin{aligned}
\dot{H}(E, V) & =\mathbf{e}^{\mathrm{T}} \dot{E}+\sum_{i=1}^{q}\left(\frac{\partial \mathcal{S}(E, V)}{\partial E_{i}}\right)^{-1}\left(\frac{\partial \mathcal{S}(E, V)}{\partial V_{i}}\right) \dot{V}_{i} \\
& =\mathbf{e}^{\mathrm{T}} \dot{E}+\sum_{i=1}^{q}\left(d_{\mathrm{w} i}(E, V)-S_{\mathrm{w} i}(t)\right) \\
& =\mathbf{e}^{\mathrm{T}} w(E, V) \\
& =0 .
\end{aligned}
$$

\section{Chemical equilibria, entropy production, and chemical thermodynamics}

In its most general form thermodynamics can also involve reacting mixtures and combustion. When a chemical reaction occurs, the bonds within molecules of the reactant are broken, and atoms and electrons rearrange to form products. The thermodynamic analysis of reactive systems can be addressed as an extension of the compartmental thermodynamic model described in Sections 3 and 4. Specifically, in this case the compartments would qualitatively represent different quantities in the same space, and the intercompartmental flows would represent transformation rates in addition to transfer rates. In particular, the compartments would additionally represent quantities of different chemical substances contained within the compartment, and the compartmental flows would additionally characterize transformation rates of reactants into products. In this case, an additional mass balance equation is included for addressing conservation of energy as well as conservation of mass. This additional mass conservation equation would involve the law of mass-action enforcing proportionality between a particular reaction rate and the concentrations of the reactants, and the law of superposition of elementary reactions assuring that the resultant rates for a particular species is the sum of the elementary reaction rates for the species.

In this section, we consider the interconnected dynamical system $\mathcal{G}$ where each subsystem represents a substance or species that can exchange energy with other substances as well as undergo chemical reactions with other substances forming products. Thus, the reactants and products of chemical reactions represent subsystems of $\mathcal{G}$ with the mechanisms of heat exchange between subsystems remaining the same as delineated in Section 3. Here, for simplicity of exposition, we do not consider work done by the subsystem on the environment nor work done by the environment on the system. This extension can be easily addressed using the formulation in Section 4.

To develop a dynamical systems framework for thermodynamics with chemical reaction networks, let $q$ be the total number of species (i.e., reactants and products), that is, the number of subsystems in $\mathcal{G}$, and let $X_{j}, j=1, \ldots, q$, denote the $j$ th species. Consider a single chemical reaction described by

$$
\sum_{j=1}^{q} A_{j} X_{j} \stackrel{k}{\longrightarrow} \sum_{j=1}^{q} B_{j} X_{j}
$$


where $A_{j}, B_{j}, j=1, \ldots, q$, are the stoichiometric coefficients and $k$ denotes the reaction rate. Note that the values of $A_{j}$ corresponding to the products and the values of $B_{j}$ corresponding to the reactants are zero. For example, for the familiar reaction

$$
2 \mathrm{H}_{2}+\mathrm{O}_{2} \stackrel{k}{\longrightarrow} 2 \mathrm{H}_{2} \mathrm{O},
$$

$X_{1}, X_{2}$, and $X_{3}$ denote the species $\mathrm{H}_{2}, \mathrm{O}_{2}$, and $\mathrm{H}_{2} \mathrm{O}$, respectively, and $A_{1}=2, A_{2}=1, A_{3}=0$, $B_{1}=0, B_{2}=0$, and $B_{3}=2$.

In general, for a reaction network consisting of $r \geq 1$ reactions, the $i$ th reaction is written as

$$
\sum_{j=1}^{q} A_{i j} X_{j} \stackrel{k_{i}}{\longrightarrow} \sum_{j=1}^{q} B_{i j} X_{j}, \quad i=1, \ldots, r,
$$

where, for $i=1, \ldots, r, k_{i}>0$ is the reaction rate of the $i$ th reaction, $\sum_{j=1}^{q} A_{i j} X_{j}$ is the reactant of the $i$ th reaction, and $\sum_{j=1}^{q} B_{i j} X_{j}$ is the product of the $i$ th reaction. Each stoichiometric coefficient $A_{i j}$ and $B_{i j}$ is a nonnegative integer. Note that each reaction in the reaction network (34) is represented as being irreversible. ${ }^{2}$ Reversible reactions can be modeled by including the reverse reaction as a separate reaction. The reaction network (34) can be written compactly in matrix-vector form as

$$
A X \stackrel{k}{\longrightarrow} B X
$$

where $X=\left[X_{1}, \ldots, X_{q}\right]^{\mathrm{T}}$ is a column vector of species, $k=\left[k_{1}, \ldots, k_{r}\right]^{\mathrm{T}} \in \mathbb{R}_{+}^{r}$ is a positive vector of reaction rates, and $A \in \mathbb{R}^{r \times q}$ and $B \in \mathbb{R}^{r \times q}$ are nonnegative matrices such that $A_{(i, j)}=A_{i j}$ and $B_{(i, j)}=B_{i j}, i=1, \ldots, r, j=1, \ldots, q$.

Let $n_{j}:[0, \infty) \rightarrow \overline{\mathbb{R}}_{+}, j=1, \ldots, q$, denote the mole number of the $j$ th species and define $n \triangleq\left[n_{1}, \ldots, n_{q}\right]^{\mathrm{T}}$. Invoking the law of mass-action (Steinfeld et al. (1989)), which states that, for an elementary reaction, that is, a reaction in which all of the stoichiometric coefficients of the reactants are one, the rate of reaction is proportional to the product of the concentrations of the reactants, the species quantities change according to the dynamics (Haddad et al. (2010); Chellaboina et al. (2009))

$$
\dot{n}(t)=(B-A)^{\mathrm{T}} K n^{A}(t), \quad n(0)=n_{0}, \quad t \geq t_{0},
$$

where $K \triangleq \operatorname{diag}\left[k_{1}, \ldots, k_{r}\right] \in \mathbb{P}^{r}$ and

$$
n^{A} \triangleq\left[\begin{array}{c}
\prod_{j=1}^{q} n_{j}^{A_{1 j}} \\
\vdots \\
\prod_{j=1}^{q} n_{j}^{A_{r j}}
\end{array}\right]=\left[\begin{array}{c}
n_{1}^{A_{11}} \cdots n_{q}^{A_{1 q}} \\
\vdots \\
n_{1}^{A_{r 1}} \cdots n_{q}^{A_{r q}}
\end{array}\right] \in \overline{\mathbb{R}}_{+}^{r} .
$$

For details regarding the law of mass-action and Equation (36), see Erdi \& Toth (1988); Haddad et al. (2010); Steinfeld et al. (1989); Chellaboina et al. (2009). Furthermore, let $M_{j}>0$,

\footnotetext{
${ }^{2}$ Irreversibility here refers to the fact that part of the chemical reaction involves generation of products from the original reactants. Reversible chemical reactions that involve generation of products from the reactants and vice versa can be modeled as two irreversible reactions; one of which involves generation of products from the reactants and the other involving generation of the original reactants from the products.
} 
$j=1, \ldots, q$, denote the molar mass (i.e., the mass of one mole of a substance) of the $j$ th species, let $m_{j}:[0, \infty) \rightarrow \overline{\mathbb{R}}_{+}, j=1, \ldots, q$, denote the mass of the $j$ th species so that $m_{j}(t)=M_{j} n_{j}(t)$, $t \geq t_{0}, j=1, \ldots, q$, and let $m \triangleq\left[m_{1}, \ldots, m_{q}\right]^{\mathrm{T}}$. Then, using the transformation $m(t)=\operatorname{Mn}(t)$, where $M \triangleq \operatorname{diag}\left[M_{1}, \ldots, M_{q}\right] \in \mathbb{P}^{q}$, (36) can be rewritten as the mass balance equation

$$
\dot{m}(t)=M(B-A)^{\mathrm{T}} \tilde{K} m^{A}(t), \quad m(0)=m_{0}, \quad t \geq t_{0},
$$

where $\tilde{K} \triangleq \operatorname{diag}\left[\frac{k_{1}}{\prod_{j=1}^{q} M_{j}^{A_{1 j}}}, \ldots, \frac{k_{r}}{\prod_{j=1}^{q} M_{j}^{A_{r j}}}\right] \in \mathbb{P}^{r}$.

In the absence of nuclear reactions, the total mass of the species during each reaction in (35) is conserved. Specifically, consider the $i$ th reaction in (35) given by (34) where the mass of the reactants is $\sum_{j=1}^{q} A_{i j} M_{j}$ and the mass of the products is $\sum_{j=1}^{q} B_{i j} M_{j}$. Hence, conservation of mass in the $i$ th reaction is characterized as

$$
\sum_{j=1}^{q}\left(B_{i j}-A_{i j}\right) M_{j}=0, \quad i=1, \ldots, r,
$$

or, in general for (35), as

$$
\mathbf{e}^{\mathrm{T}} M(B-A)^{\mathrm{T}}=0 .
$$

Note that it follows from (38) and (40) that $\mathbf{e}^{\mathrm{T}} \dot{m}(t) \equiv 0$.

Equation (38) characterizes the change in masses of substances in the interconnected dynamical system $\mathcal{G}$ due to chemical reactions. In addition to the change of mass due to chemical reactions, each substance can exchange energy with other substances according to the energy flow mechanism described in Section 3; that is, energy flows from substances at a higher temperature to substances at a lower temperature. Furthermore, in the presence of chemical reactions, the exchange of matter affects the change of energy of each substance through the quantity known as the chemical potential.

The notion of the chemical potential was introduced by Gibbs in 1875-1878 (Gibbs (1875; 1878)) and goes far beyond the scope of chemistry effecting virtually every process in nature (Baierlein (2001); Fuchs (1996); Job \& Herrmann (2006)). The chemical potential has a strong connection with the second law of thermodynamics in that every process in nature evolves from a state of higher chemical potential towards a state of lower chemical potential. It was postulated by Gibbs $(1875 ; 1878)$ that the change in energy of a homogeneous substance is proportional to the change in mass of this substance with the coefficient of proportionality given by the chemical potential of the substance.

To elucidate this, assume the $j$ th substance corresponds to the $j$ th compartment and consider the rate of energy change of the $j$ th substance of $\mathcal{G}$ in the presence of matter exchange. In this case, it follows from (5) and Gibbs' postulate that the rate of energy change of the $j$ th substance is given by

$$
\begin{aligned}
& \dot{E}_{j}(t)=\left[\sum_{k=1, k \neq j}^{q} \phi_{j k}(E(t))\right]-\sigma_{j j}(E(t))+S_{j}(t)+\mu_{j}(E(t), m(t)) \dot{m}_{j}(t), \quad E_{j}\left(t_{0}\right)=E_{j 0}, \\
& t \geq t_{0},
\end{aligned}
$$

where $\mu_{j}: \overline{\mathbb{R}}_{+}^{q} \times \overline{\mathbb{R}}_{+}^{q} \rightarrow \mathbb{R}, j=1, \ldots, q$, is the chemical potential of the $j$ th substance. It follows from (41) that $\mu_{j}(\cdot, \cdot)$ is the chemical potential of a unit mass of the $j$ th substance. We assume 
that if $E_{j}=0$, then $\mu_{j}(E, m)=0, j=1, \ldots, q$, which implies that if the energy of the $j$ th substance is zero, then its chemical potential is also zero.

Next, using (38) and (41), the energy and mass balance equations for the interconnected dynamical system $\mathcal{G}$ can be written as

$$
\begin{aligned}
& \dot{E}(t)=w(E(t))+P(E(t), m(t)) M(B-A)^{\mathrm{T}} \tilde{K} m^{A}(t)-d(E(t))+S(t), \quad \begin{aligned}
E\left(t_{0}\right)=E_{0} \\
t \geq t_{0},
\end{aligned} \\
& \dot{m}(t)=M(B-A)^{\mathrm{T}} \tilde{K} m^{A}(t), \quad m(0)=m_{0}
\end{aligned}
$$

where $P(E, m) \triangleq \operatorname{diag}\left[\mu_{1}(E, m), \ldots, \mu_{q}(E, m)\right] \in \mathbb{R}^{q \times q}$ and where $w(\cdot), d(\cdot)$, and $S(\cdot)$ are defined as in Section 3. It follows from Proposition 1 of Chellaboina et al. (2009) that the dynamics of (43) are essentially nonnegative and, since $\mu_{j}(E, m)=0$ if $E_{j}=0, j=1, \ldots, q$, it also follows that, for the isolated dynamical system $\mathcal{G}$ (i.e., $S(t) \equiv 0$ and $d(E) \equiv 0$ ), the dynamics of (42) and (43) are essentially nonnegative.

Note that, for the $i$ th reaction in the reaction network (35), the chemical potentials of the reactants and the products are $\sum_{j=1}^{q} A_{i j} M_{j} \mu_{j}(E, m)$ and $\sum_{j=1}^{q} B_{i j} M_{j} \mu_{j}(E, m)$, respectively. Thus,

$$
\sum_{j=1}^{q} B_{i j} M_{j} \mu_{j}(E, m)-\sum_{j=1}^{q} A_{i j} M_{j} \mu_{j}(E, m) \leq 0, \quad(E, m) \in \overline{\mathbb{R}}_{+}^{q} \times \overline{\mathbb{R}}_{+}^{q},
$$

is a restatement of the principle that a chemical reaction evolves from a state of a greater chemical potential to that of a lower chemical potential, which is consistent with the second law of thermodynamics. The difference between the chemical potential of the reactants and the chemical potential of the products is called affinity (DeDonder (1927); DeDonder \& Rysselberghe (1936)) and is given by

$$
v_{i}(E, m)=\sum_{j=1}^{q} A_{i j} M_{j} \mu_{j}(E, m)-\sum_{j=1}^{q} B_{i j} M_{j} \mu_{j}(E, m) \geq 0, \quad i=1, \ldots, r .
$$

Affinity is a driving force for chemical reactions and is equal to zero at the state of chemical equilibrium. A nonzero affinity implies that the system in not in equilibrium and that chemical reactions will continue to occur until the system reaches an equilibrium characterized by zero affinity. The next assumption provides a general form for the inequalities (44) and (45).

Assumption 5.1 For the chemical reaction network (35) with the mass balance equation (43), assume that $\mu(E, m)>>0$ for all $E \neq 0$ and

$$
(B-A) M \mu(E, m) \leq \leq 0, \quad(E, m) \in \overline{\mathbb{R}}_{+}^{q} \times \overline{\mathbb{R}}_{+}^{q},
$$

or, equivalently,

$$
v(E, m)=(A-B) M \mu(E, m) \geq \geq 0, \quad(E, m) \in \overline{\mathbb{R}}_{+}^{q} \times \overline{\mathbb{R}}_{+,}^{q}
$$

where $\mu(E, m) \triangleq\left[\mu_{1}(E, m), \ldots, \mu_{q}(E, m)\right]^{\mathrm{T}}$ is the vector of chemical potentials of the substances of $\mathcal{G}$ and $\nu(E, m) \triangleq\left[v_{1}(E, m), \ldots, v_{r}(E, m)\right]^{\mathrm{T}}$ is the affinity vector for the reaction network (35). 
Note that equality in (46) or, equivalently, in (47) characterizes the state of chemical equilibrium when the chemical potentials of the products and reactants are equal or, equivalently, when the affinity of each reaction is equal to zero. In this case, no reaction occurs and $\dot{m}(t)=0, t \geq t_{0}$.

Next, we characterize the entropy function for the interconnected dynamical system $\mathcal{G}$ with the energy and mass balance equations (42) and (43). The definition of entropy for $\mathcal{G}$ in the presence of chemical reactions remains the same as in Definition 3.1 with $\mathcal{S}(E)$ replaced by $\mathcal{S}(E, m)$ and with all other conditions in the definition holding for every $m>>0$. Consider the $j$ th subsystem of $\mathcal{G}$ and assume that $E_{k}$ and $m_{k}, k \neq j, k=1, \ldots, q$, are constant. In this case, note that

$$
\frac{\mathrm{d} \mathcal{S}}{\mathrm{d} t}=\frac{\partial \mathcal{S}}{\partial E_{j}} \frac{\mathrm{d} E_{j}}{\mathrm{~d} t}+\frac{\partial \mathcal{S}}{\partial m_{j}} \frac{\mathrm{d} m_{j}}{\mathrm{~d} t}
$$

and recall that

$$
\frac{\partial \mathcal{S}}{\partial E} P(E, m)+\frac{\partial \mathcal{S}}{\partial m}=0
$$

Next, it follows from (49) that the time derivative of the entropy function $\mathcal{S}(E, m)$ along the trajectories of (42) and (43) is given by

$$
\begin{aligned}
& \dot{\mathcal{S}}(E, m)= \frac{\partial \mathcal{S}(E, m)}{\partial E} \dot{E}+\frac{\partial \mathcal{S}(E, m)}{\partial m} \dot{m} \\
&= \frac{\partial \mathcal{S}(E, m)}{\partial E} w(E)+\left(\frac{\partial \mathcal{S}(E, m)}{\partial E} P(E, m)+\frac{\partial \mathcal{S}(E, m)}{\partial m}\right) M(B-A)^{\mathrm{T}} \tilde{K} m^{A} \\
&+\frac{\partial \mathcal{S}(E, m)}{\partial E} S(t)-\frac{\partial \mathcal{S}(E, m)}{\partial E} d(E) \\
&= \frac{\partial \mathcal{S}(E, m)}{\partial E} w(E)+\frac{\partial \mathcal{S}(E, m)}{\partial E} S(t)-\frac{\partial \mathcal{S}(E, m)}{\partial E} d(E) \\
&= \sum_{i=1}^{q} \sum_{j=i+1}^{q}\left(\frac{\partial \mathcal{S}(E, m)}{\partial E_{i}}-\frac{\partial \mathcal{S}(E, m)}{\partial E_{j}}\right) \phi_{i j}(E)+\frac{\partial \mathcal{S}(E, m)}{\partial E} S(t)-\frac{\partial \mathcal{S}(E, m)}{\partial E} d(E), \\
& \quad(E, m) \in \overline{\mathbb{R}}_{+}^{q} \times \overline{\mathbb{R}}_{+}^{q} .
\end{aligned}
$$

For the isolated system $\mathcal{G}$ (i.e., $S(t) \equiv 0$ and $d(E) \equiv 0$ ), the entropy function of $\mathcal{G}$ is a nondecreasing function of time and, using identical arguments as in the proof of Theorem 3.1, it can be shown that $(E(t), m(t)) \rightarrow \mathcal{R} \triangleq\left\{(E, m) \in \overline{\mathbb{R}}_{+}^{q} \times \overline{\mathbb{R}}_{+}^{q}: \frac{\partial \mathcal{S}(E, m)}{\partial E_{1}}=\cdots=\frac{\partial \mathcal{S}(E, m)}{\partial E_{q}}\right\}$ as $t \rightarrow \infty$ for all $\left(E_{0}, m_{0}\right) \in \overline{\mathbb{R}}_{+}^{q} \times \overline{\mathbb{R}}_{+}^{q}$.

The entropy production in the interconnected system $\mathcal{G}$ due to chemical reactions is given by

$$
\begin{aligned}
\mathrm{d} \mathcal{S}_{\mathrm{i}}(E, m) & =\frac{\partial \mathcal{S}(E, m)}{\partial m} \mathrm{~d} m \\
& =-\frac{\partial \mathcal{S}(E, m)}{\partial E} P(E, m) M(B-A)^{\mathrm{T}} \tilde{K} m^{A} \mathrm{~d} t, \quad(E, m) \in \overline{\mathbb{R}}_{+}^{q} \times \overline{\mathbb{R}}_{+}^{q} .
\end{aligned}
$$

If the interconnected dynamical system $\mathcal{G}$ is isothermal, that is, all subsystems of $\mathcal{G}$ are at the same temperature

$$
\left(\frac{\partial \mathcal{S}(E, m)}{\partial E_{1}}\right)^{-1}=\cdots=\left(\frac{\partial \mathcal{S}(E, m)}{\partial E_{q}}\right)^{-1}=T,
$$


where $T>0$ is the system temperature, then it follows from Assumption 5.1 that

$$
\begin{aligned}
\mathrm{d} \mathcal{S}_{\mathrm{i}}(E, m) & =-\frac{1}{T} \mathbf{e}^{\mathrm{T}} P(E, m) M(B-A)^{\mathrm{T}} \tilde{\mathrm{K}} m^{A} \mathrm{~d} t \\
& =-\frac{1}{T} \mu^{\mathrm{T}}(E, m) M(B-A)^{\mathrm{T}} \tilde{\mathrm{K}} m^{A} \mathrm{~d} t \\
& =\frac{1}{T} v^{\mathrm{T}}(E, m) \tilde{K} m^{A} \mathrm{~d} t \\
& \geq 0, \quad(E, m) \in \overline{\mathbb{R}}_{+}^{q} \times \overline{\mathbb{R}}_{+}^{q} .
\end{aligned}
$$

Note that since the affinity of a reaction is equal to zero at the state of a chemical equilibrium, it follows that equality in (53) holds if and only if $v(E, m)=0$ for some $E \in \overline{\mathbb{R}}_{+}^{q}$ and $m \in \overline{\mathbb{R}}_{+}^{q}$.

Theorem 5.1 Consider the isolated (i.e., $S(t) \equiv 0$ and $d(E) \equiv 0$ ) interconnected dynamical system $\mathcal{G}$ with the power and mass balance equations (42) and (43). Assume that rankC $=q-1$, Assumption 5.1 holds, and there exists an entropy function $\mathcal{S}: \overline{\mathbb{R}}_{+}^{q} \times \overline{\mathbb{R}}_{+}^{q} \rightarrow \mathbb{R}$ of $\mathcal{G}$. Then $(E(t), m(t)) \rightarrow \mathcal{R}$ as $t \rightarrow \infty$, where $(E(t), m(t)), t \geq t_{0}$, is the solution to (42) and (43) with the initial condition $\left(E_{0}, m_{0}\right) \in$ $\overline{\mathbb{R}}_{+}^{q} \times \overline{\mathbb{R}}_{+}^{q}$ and

$$
\mathcal{R}=\left\{(E, m) \in \overline{\mathbb{R}}_{+}^{q} \times \overline{\mathbb{R}}_{+}^{q}: \frac{\partial \mathcal{S}(E, m)}{\partial E_{1}}=\cdots=\frac{\partial \mathcal{S}(E, m)}{\partial E_{q}} \text { and } v(E, m)=0\right\},
$$

where $v(\cdot, \cdot)$ is the affinity vector of $\mathcal{G}$.

Proof. Since the dynamics of the isolated system $\mathcal{G}$ are essentially nonnegative, it follows from Proposition 21 that $(E(t), m(t)) \in \overline{\mathbb{R}}_{+}^{q} \times \overline{\mathbb{R}}_{+}^{q}, t \geq t_{0}$, for all $\left(E_{0}, m_{0}\right) \in \overline{\mathbb{R}}_{+}^{q} \times \overline{\mathbb{R}}_{+}^{q}$. Consider a scalar function $v(E, m)=\mathbf{e}^{\mathrm{T}} E+\mathbf{e}^{\mathrm{T}} m,(E, m) \in \overline{\mathbb{R}}_{+}^{q} \times \overline{\mathbb{R}}_{+}^{q}$, and note that $v(0,0)=0$ and $v(E, m)>$ $0,(E, m) \in \overline{\mathbb{R}}_{+}^{q} \times \overline{\mathbb{R}}_{+}^{q},(E, m) \neq(0,0)$. It follows from (40), Assumption 5.1, and $\mathbf{e}^{\mathrm{T}} w(E) \equiv 0$ that the time derivative of $v(\cdot, \cdot)$ along the trajectories of (42) and (43) satisfies

$$
\begin{aligned}
\dot{v}(E, m) & =\mathbf{e}^{\mathrm{T}} \dot{E}+\mathbf{e}^{\mathrm{T}} \dot{m} \\
& =\mathbf{e}^{\mathrm{T}} P(E, m) M(B-A)^{\mathrm{T}} \tilde{K} m^{A} \\
& =\mu^{\mathrm{T}}(E, m) M(B-A)^{\mathrm{T}} \tilde{K} m^{A} \\
& =-v^{\mathrm{T}}(E, m) \tilde{K} m^{A} \\
& \leq 0, \quad(E, m) \in \overline{\mathbb{R}}_{+}^{q} \times \overline{\mathbb{R}}_{+}^{q},
\end{aligned}
$$

which implies that the solution $(E(t), m(t)), t \geq t_{0}$, to (42) and (43) is bounded for all initial conditions $\left(E_{0}, m_{0}\right) \in \overline{\mathbb{R}}_{+}^{q} \times \overline{\mathbb{R}}_{+}^{q}$.

Next, consider the function $\tilde{v}(E, m)=\mathbf{e}^{\mathrm{T}} E+\mathbf{e}^{\mathrm{T}} m-\mathcal{S}(E, m),(E, m) \in \overline{\mathbb{R}}_{+}^{q} \times \overline{\mathbb{R}}_{+}^{q}$. Then it follows from (50) and (55) that the time derivative of $\tilde{v}(\cdot, \cdot)$ along the trajectories of (42) and (43) satisfies

$$
\begin{aligned}
\dot{\tilde{v}}(E, m) & =\mathbf{e}^{\mathrm{T}} \dot{E}+\mathbf{e}^{\mathrm{T}} \dot{m}-\dot{\mathcal{S}}(E, m) \\
& =-v^{\mathrm{T}}(E, m) \tilde{K} m^{A}-\sum_{i=1}^{q} \sum_{j=i+1}^{q}\left(\frac{\partial \mathcal{S}(E, m)}{\partial E_{i}}-\frac{\partial \mathcal{S}(E, m)}{\partial E_{j}}\right) \phi_{i j}(E) \\
& \leq 0, \quad(E, m) \in \overline{\mathbb{R}}_{+}^{q} \times \overline{\mathbb{R}}_{+}^{q}
\end{aligned}
$$


which implies that $\tilde{v}(\cdot, \cdot)$ is a nonincreasing function of time, and hence, by the Krasovskii-LaSalle theorem (Haddad \& Chellaboina (2008)), $(E(t), m(t)) \rightarrow \mathcal{R} \triangleq\{(E, m) \in$ $\left.\overline{\mathbb{R}}_{+}^{q} \times \overline{\mathbb{R}}_{+}^{q}: \dot{\tilde{v}}(E, m)=0\right\}$ as $t \rightarrow \infty$. Now, it follows from Definition 3.1, Assumption 5.1, and the fact that $\operatorname{rank} \mathcal{C}=q-1$ that

$$
\begin{aligned}
\mathcal{R}= & \left\{(E, m) \in \overline{\mathbb{R}}_{+}^{q} \times \overline{\mathbb{R}}_{+}^{q}: \frac{\partial \mathcal{S}(E, m)}{\partial E_{1}}=\cdots=\frac{\partial \mathcal{S}(E, m)}{\partial E_{q}}\right\} \\
& \cap\left\{(E, m) \in \overline{\mathbb{R}}_{+}^{q} \times \overline{\mathbb{R}}_{+}^{q}: v(E, m)=0\right\},
\end{aligned}
$$

which proves the result.

Theorem 5.1 implies that the state of the interconnected dynamical system $\mathcal{G}$ converges to the state of thermal and chemical equilibrium when the temperatures of all substances of $\mathcal{G}$ are equal and the masses of all substances reach a state where all reaction affinities are zero corresponding to a halting of all chemical reactions.

Next, we assume that the entropy of the interconnected dynamical system $\mathcal{G}$ is a sum of individual entropies of subsystems of $\mathcal{G}$, that is, $\mathcal{S}(E, m)=\sum_{j=1}^{q} \mathcal{S}_{j}\left(E_{j}, m_{j}\right),(E, m) \in \overline{\mathbb{R}}_{+}^{q} \times \overline{\mathbb{R}}_{+}^{q}$. In this case, the Helmholtz free energy of $\mathcal{G}$ is given by

$$
F(E, m)=\mathbf{e}^{\mathrm{T}} E-\sum_{j=1}^{q}\left(\frac{\partial \mathcal{S}(E, m)}{\partial E_{j}}\right)^{-1} \mathcal{S}_{j}\left(E_{j}, m_{j}\right), \quad(E, m) \in \overline{\mathbb{R}}_{+}^{q} \times \overline{\mathbb{R}}_{+}^{q} .
$$

If the interconnected dynamical system $\mathcal{G}$ is isothermal, then the derivative of $F(\cdot, \cdot)$ along the trajectories of (42) and (43) is given by

$$
\begin{aligned}
\dot{F}(E, m) & =\mathbf{e}^{\mathrm{T}} \dot{E}-\sum_{j=1}^{q}\left(\frac{\partial \mathcal{S}(E, m)}{\partial E_{j}}\right)^{-1} \dot{\mathcal{S}}_{j}\left(E_{j}, m_{j}\right) \\
& =\mathbf{e}^{\mathrm{T}} \dot{E}-\sum_{j=1}^{q}\left(\frac{\partial \mathcal{S}(E, m)}{\partial E_{j}}\right)^{-1}\left[\frac{\partial \mathcal{S}_{j}\left(E_{j}, m_{j}\right)}{\partial E_{j}} \dot{E}_{j}+\frac{\partial \mathcal{S}_{j}\left(E_{j}, m_{j}\right)}{\partial m_{j}} \dot{m}_{j}\right] \\
& =\mu^{\mathrm{T}}(E, m) M(B-A)^{\mathrm{T}} \tilde{K} m^{A} \\
& =-v^{\mathrm{T}}(E, m) \tilde{K} m^{A} \\
& \leq 0, \quad(E, m) \in \overline{\mathbb{R}}_{+}^{q} \times \overline{\mathbb{R}}_{+}^{q}
\end{aligned}
$$

with equality in (59) holding if and only if $v(E, m)=0$ for some $E \in \overline{\mathbb{R}}_{+}^{q}$ and $m \in \overline{\mathbb{R}}_{+}^{q}$, which determines the state of chemical equilibrium. Hence, the Helmholtz free energy of $\mathcal{G}$ evolves to a minimum when the pressure and temperature of each subsystem of $\mathcal{G}$ are maintained constant, which is consistent with classical thermodynamics. A similar conclusion can be arrived at for the Gibbs free energy if work energy considerations to and by the system are addressed. Thus, the Gibbs and Helmholtz free energies are a measure of the tendency for a reaction to take place in the interconnected system $\mathcal{G}$, and hence, provide a measure of the work done by the interconnected system $\mathcal{G}$.

\section{Conclusion}

In this paper, we developed a system-theoretic perspective for classical thermodynamics and chemical reaction processes. In particular, we developed a nonlinear compartmental 
model involving heat flow, work energy, and chemical reactions that captures all of the key aspects of thermodynamics, including its fundamental laws. In addition, we showed that the interconnected compartmental model gives rise to globally semistable equilibria involving states of temperature equipartition. Finally, using the notion of the chemical potential, we combined our heat flow compartmental model with a state space mass-action kinetics model to capture energy and mass exchange in interconnected large-scale systems in the presence of chemical reactions. In this case, it was shown that the system states converge to a state of temperature equipartition and zero affinity.

\section{References}

Arnold, V. (1990). Contact geometry: The geometrical method of Gibbs' thermodynamics, in D. Caldi \& G. Mostow (eds), Proceedings of the Gibbs Symposium, American Mathematical Society, Providence, RI, pp. 163-179.

Baierlein, R. (2001). The elusive chemical potential, Amer. J. Phys. 69(4): 423-434.

Chellaboina, V., Bhat, S. P., Haddad, W. M. \& Bernstein, D. S. (2009). Modeling and analysis of mass action kinetics: Nonnegativity, realizability, reducibility, and semistability, Contr. Syst. Mag. 29(4): 60-78.

DeDonder, T. (1927). L'Affinité, Gauthiers-Villars, Paris.

DeDonder, T. \& Rysselberghe, P. V. (1936). Affinity, Stanford University Press, Menlo Park, CA.

Diestel, R. (1997). Graph Theory, Springer-Verlag, New York, NY.

Erdi, P. \& Toth, J. (1988). Mathematical Models of Chemical Reactions: Theory and Applications of Deterministic and Stochastic Models, Princeton University Press, Princeton, NJ.

Fuchs, H. U. (1996). The Dynamics of Heat, Springer-Verlag, New York, NY.

Gibbs, J. W. (1875). On the equilibrium of heterogeneous substances, Tras. Conn. Acad. Sci. III: $108-248$.

Gibbs, J. W. (1878). On the equilibrium of heterogeneous substances, Trans. Conn. Acad. Sci. III: $343-524$.

Godsil, C. \& Royle, G. (2001). Algebraic Graph Theory, Springer-Verlag, New York.

Haddad, W. M. \& Chellaboina, V. (2005). Stability and dissipativity theory for nonnegative dynamical systems: A unified analysis framework for biological and physiological systems, Nonlinear Analysis: Real World Applications 6: 35-65.

Haddad, W. M. \& Chellaboina, V. (2008). Nonlinear Dynamical Systems and Control. A Lyapunov-Based Approach, Princeton University Press, Princeton, NJ.

Haddad, W. M., Chellaboina, V. \& Hui, Q. (2010). Nonnegative and Compartmental Dynamical Systems, Princeton University Press, Princeton, NJ.

Haddad, W. M., Chellaboina, V. \& Nersesov, S. G. (2005). Thermodynamics. A Dynamical Systems Approach, Princeton University Press, Princeton, NJ.

Haddad, W. M., Chellaboina, V. \& Nersesov, S. G. (2008). Time-reversal symmetry, Poincaré recurrence, irreversibility, and the entropic arrow of time: From mechanics to system thermodynamics, Nonlinear Analysis: Real World Applications 9: 250-271.

Hartman, P. (1982). Ordinary Differential Equations, Birkhäuser, Boston.

Job, G. \& Herrmann, F. (2006). Chemical potential - a quantity in search of recognition, Eur. J. Phys. 27: 353-371.

Steinfeld, J. I., Francisco, J. S. \& Hase, W. L. (1989). Chemical Kinetics and Dynamics, Prentice-Hall, Upper Saddle River, NJ.

Truesdell, C. (1969). Rational Thermodynamics, McGraw-Hill, New York, NY. 
Truesdell, C. (1980). The Tragicomical History of Thermodynamics 1822-1854, Springer-Verlag, New York, NY. 


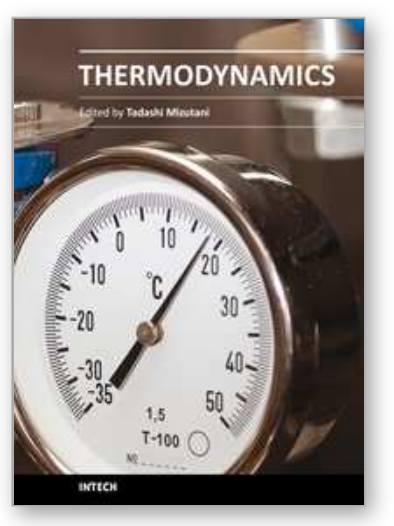

\author{
Thermodynamics \\ Edited by Prof. Mizutani Tadashi
}

ISBN 978-953-307-544-0

Hard cover, 440 pages

Publisher InTech

Published online 14, January, 2011

Published in print edition January, 2011

Progress of thermodynamics has been stimulated by the findings of a variety of fields of science and technology. The principles of thermodynamics are so general that the application is widespread to such fields as solid state physics, chemistry, biology, astronomical science, materials science, and chemical engineering. The contents of this book should be of help to many scientists and engineers.

\title{
How to reference
}

In order to correctly reference this scholarly work, feel free to copy and paste the following:

Wassim M. Haddad, Sergey G. Nersesov and VijaySekhar Chellaboina (2011). Heat Flow, Work Energy, Chemical Reactions, and Thermodynamics: a Dynamical Systems Perspective, Thermodynamics, Prof. Mizutani Tadashi (Ed.), ISBN: 978-953-307-544-0, InTech, Available from:

http://www.intechopen.com/books/thermodynamics/heat-flow-work-energy-chemical-reactions-andthermodynamics-a-dynamical-systems-perspective

\section{INTECH}

open science | open minds

\author{
InTech Europe \\ University Campus STeP Ri \\ Slavka Krautzeka 83/A \\ 51000 Rijeka, Croatia \\ Phone: +385 (51) 770447 \\ Fax: +385 (51) 686166 \\ www.intechopen.com
}

\author{
InTech China \\ Unit 405, Office Block, Hotel Equatorial Shanghai \\ No.65, Yan An Road (West), Shanghai, 200040, China \\ 中国上海市延安西路65号上海国际贵都大饭店办公楼405单元 \\ Phone: +86-21-62489820 \\ Fax: +86-21-62489821
}


(C) 2011 The Author(s). Licensee IntechOpen. This chapter is distributed under the terms of the Creative Commons Attribution-NonCommercialShareAlike-3.0 License, which permits use, distribution and reproduction for non-commercial purposes, provided the original is properly cited and derivative works building on this content are distributed under the same license. 